\title{
Mechanical Production of Very Thin Oscillator Plates
}

\author{
By Leland T. Sogn and Walter J. Howard
}

\begin{abstract}
To produce quartz oscillator plates thinner than 0.005 inch, it has been necessary to modify the usual lapping methods and machinery. The thickness at which the crystal carrier (nest) stretches or buckles is the limiting factor in the conventional apparatus. The modifications described in this report consist of replacements for the conventional top lapping plate with corresponding changes in the crystal carrier. The development is presented in chronological order from the simple pressure block method to the more elaborate forms using automatic truing. The improved equipment is capable of producing 0.001-inchthick quartz crystals and can be used for producing equally thin wafers from a variety of other materials.
\end{abstract}

\section{Introduction}

In order to manufacture crystals that are thin enough and have the proper contour to give the high fundamental frequencies now being sought, it has been necessary to modify the usual lapping procedure and to design appropriate apparatus. Various modifications have been tested; the most desirable features have been retained and obvious weaknesses corrected (as far as possible) in evolving each subsequent model. The apparently defective units have not been abandoned; reexaminations and alterations in the light of added experience have been made whenever time and funds have permitted. The most successful machines developed in this laboratory produce crystals 0.001 in. thick. Further improvements are expected, and the ultimate thickness will depend more on problems related to the handling of such fragile crystals than on the mechanical limitations of the finishing equipment.

\section{Conventional Method}

Usually blanks to be lapped are placed in the apertures of a metal or plastic nest, which carries them through a curved path between the lapstwo heavy flat plates charged with abrasive. A modified drill press drives the nest by means of an adjustable eccentric and a bushing (fig. 1). Since the nest must be thinner than the crystals to permit their abrasion, the thickness of the nest is the limiting factor. Because of the stretching and buckling that occur, this limit is about $0.005 \mathrm{in}$. for most available materials, and quartz crystals

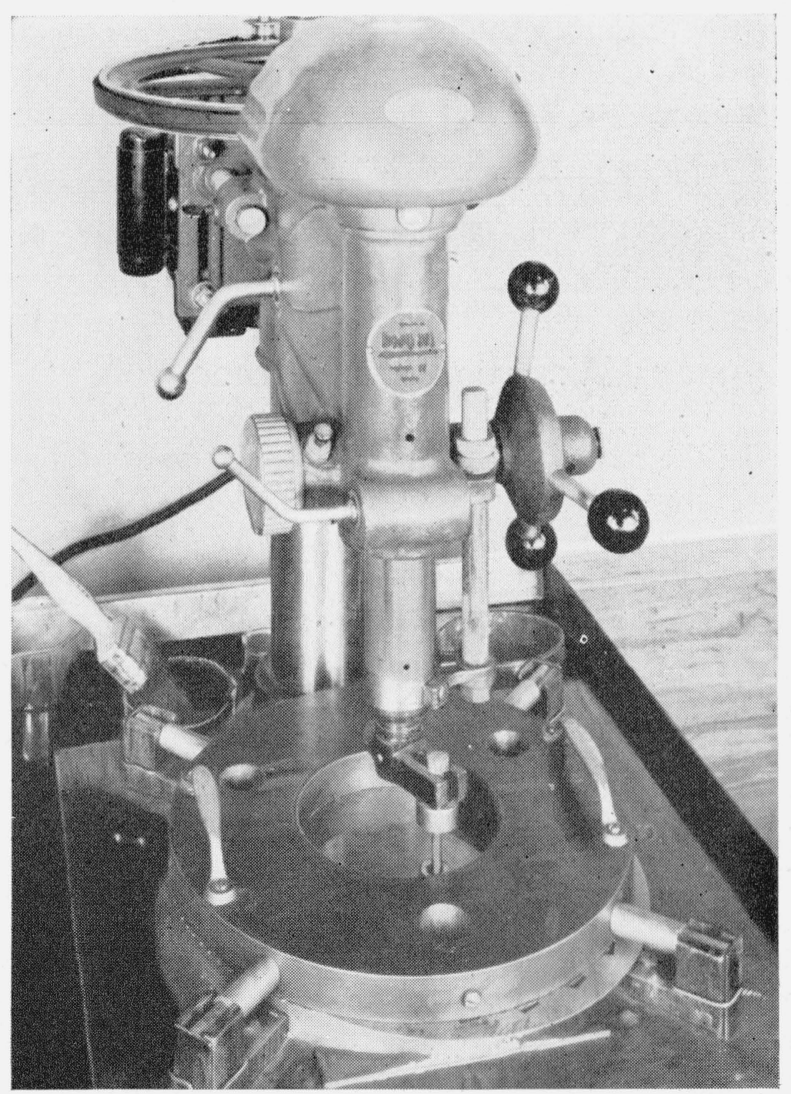

Figure 1. Conventional lapping equipment.

The thin carrier (nest) with its pentagonal openings may be seen projecting from its position between the upper and lower laps.

with such a thickness have maximum fundamental frequencies of not more than 14 and $20 \mathrm{mc}$ for $\mathrm{AT}$ and BT cuts, respectively. These cuts are most generally used for high-frequency oscillators. 


\section{Cemented Block Method}

The final thickness of the crystal was made independent of the nest thickness by putting the plane of pressure application below the upper surface of the nest. In the first modification this was accomplished by cementing a $3 / 8$-in.-high by $1 / 2$-in.square steel block to each $1 / 2$-in.-square crystal. The cemented units were placed in a nest $(0.025$ in. thick) and driven in the usual curved path over the lower lapping plate only, the individual blocks furnishing pressure previously supplied by the top plate. Blanks of less than 0.002-in. thickness were produced in this manner, but they were too wedgeshaped to oscillate.

This method of mounting had important disadvantages. Each crystal had to be mounted parallel to the block edges, without strain, precisely in the center of the base with the normal to the geometric center of the major crystal surface passing through the geometric center of the block. Such conditions were difficult to more than approximate. Furthermore, it was necessary to detach the crystal to check thickness, contour, and frequency. For these reasons other schemes were tried.

\section{Free Block Method}

In the next experiment the same apparatus was used, but the block was permitted to rest freely on the crystal (fig. 2). Slight movements of the block relative to the crystal constantly redistributed the abrasive mixture between the block and crystal, thus preventing accumulations that would cause unequal distribution of pressure by the block. However, wedge-shaped crystals again resulted and crystals tended to slip under the nest, so this procedure was suspended while other paths were pursued.

During the reinvestigation it was observed that the movements of both blocks and crystals were violent and irregular at the lowest pulley speed, so the motor speed was reduced by means of a variable autotransformer until, at $40 \mathrm{rpm}$, the motion appeared to be satisfactory. Regularly, at quarter- or half-hour intervals each block was turned $90^{\circ}$ with respect to its crystal in order to compensate for minor variations, such as the overhand of the block. Crystals 0.011 in. in thickness were brought down to 0.0025 in. without breakage, using the relatively coarse size 400 silicon carbide.

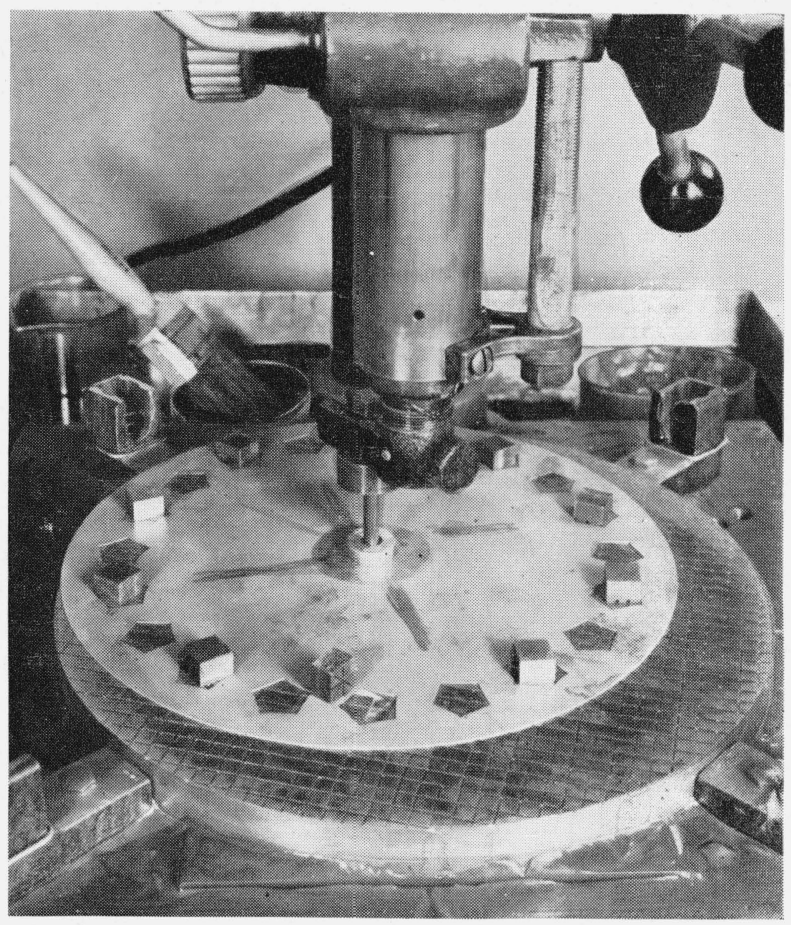

Figure 2. Free block apparatus.

Here a block of the foreground has been turned up to show both the channeling on its undersurface and the crystal that is normally beneath it. The upper surface of the lower lap is also channeled to reduce sticking.

To keep crystals from slipping beneath the thin (0.020-in.) zinc nest, small weights were placed around the loaded openings. A heavier nest would have made this unnecessary.

Crystals so processed were almost flat, being only slightly and equally high at the corners. The high corners were removed by edge-grinding to a lesser dimension.

Major advantages of the method are: The apparatus is simple; the lapping is so gentle that breakage is virtually eliminated; individual crystals may be removed from the nest easily, permitting more frequent and more satisfactory inspection; crystals of widely varying thicknesses may be simultaneously lapped. On the other hand, the rate of lapping is slow, and the crystal faces must be parallel at the start, as the block is resting on and parallel to the upper surface of the crystal rather than to the lapping plate.

\section{Square Block and Cell Method}

As a means of more effectively controlling the movement of the crystal and pressure block relative to each other, both were confined in the $1 / 2$-in. 


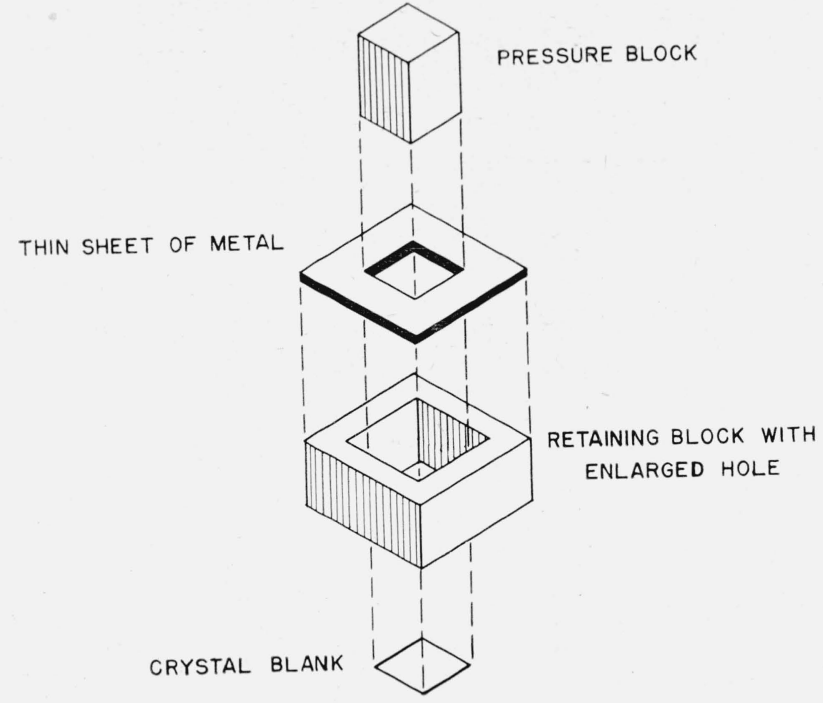

Figure 3. Square block and cell-parts.

This exploded diagram shows the relation of the various parts to each other. In operation, the pressure block rests on the crystal and passes through the thin sheet that is cemented to the retaining block.

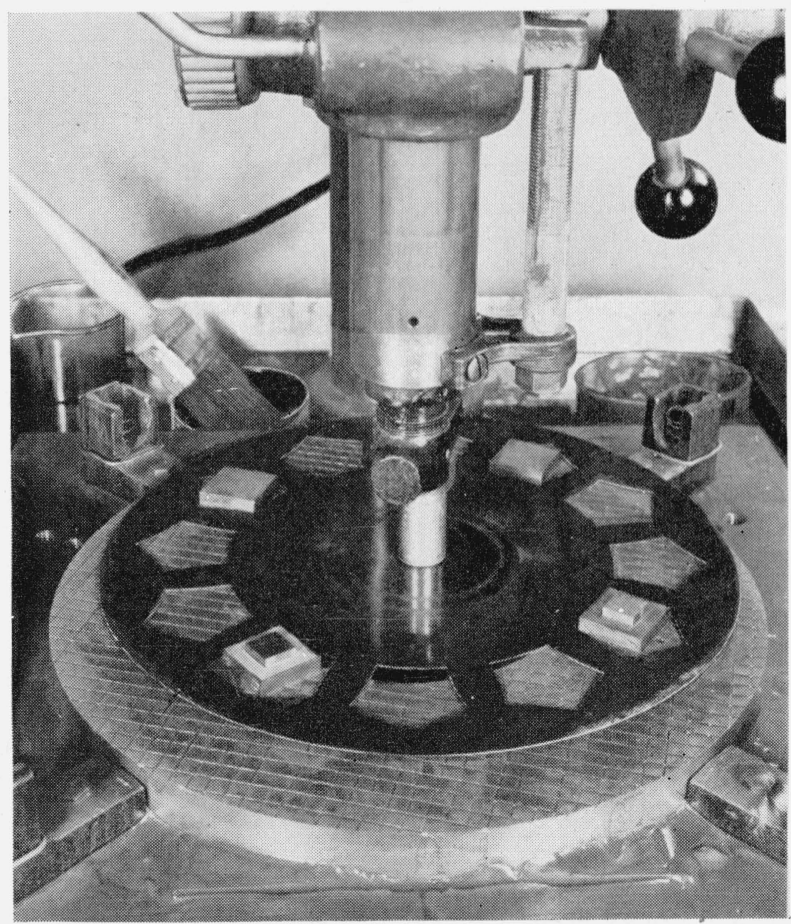

Figure 4. Square tlock and cell apparatus assembled.

Here the parts shown in the previous figure have been assembled and are ready to be driven by the nest over the lap. Blocks in background are counterweights.

square opening made through the center of a 1 -in. square by $1 / 4$-in.-thick steel plate. This unit was then placed in a nest that had pentagonal openings suitable for lapping inch square blanks. Counterweights were placed in the other nest openings to give more uniform motion.

Because the abrasive that worked into the narrow clearances between the block and plate caused binding, the opening in the plate was enlarged to about $5 / 8$ in. square. A thin zinc sheet with a $1 / 2$-in. square hole was cemented to its upper surface to hold the pressure block in position while permitting the crystal to move relative to it (fig. 3). Channels across the block surface facilitated this movement by reducing the area of adhesion between it and the crystal. This modified cell assembly was lapped in the same nest and manner used above as shown in figure 4 . Crystals produced by this method were wedgeshaped. However, the process seemed to hold so much promise that a modification that eliminated binding and permitted automatic truing was designed. This unit, which employs a cylinder and plunger, has been put into operation recently. Its performance is described later in the article.

\section{Pentagonal Block and Ring Method}

In a somewhat more elaborate apparatus (fig. 5 ), pentagonal blocks were rigidly attached to a lapped ring. The blocks fitted snugly in the openings of the nest, which was driven by the eccentric as before. Because this arrangement permitted truing of the pentagonal blocks until the lower surfaces were coplanar and parallel to the lap, wedge-shaped crystals could be corrected to parallelism, an important feature. Adhesion between blanks and blocks was reduced by channeling the under surface of the blocks. Springs between the ring and nest forced the nest into closer contact with the lap, thereby eliminating the breakage due to crystals slipping beneath the nest. Weights could be used in place of springs but have not yet been tried.

In this method, as in all the modified procedures, the greater part of the abrasion took place on the under surface of the crystals, the top surface being more polished than abraded. To insure equal wear and similar contours on both surfaces, the crystals were inverted at regular intervals. Since the contours of the crystals were dependent on the trueness of the pentagonal blocks, the blocks (still attached to the ring) were lapped before and at suitable intervals during each run. 


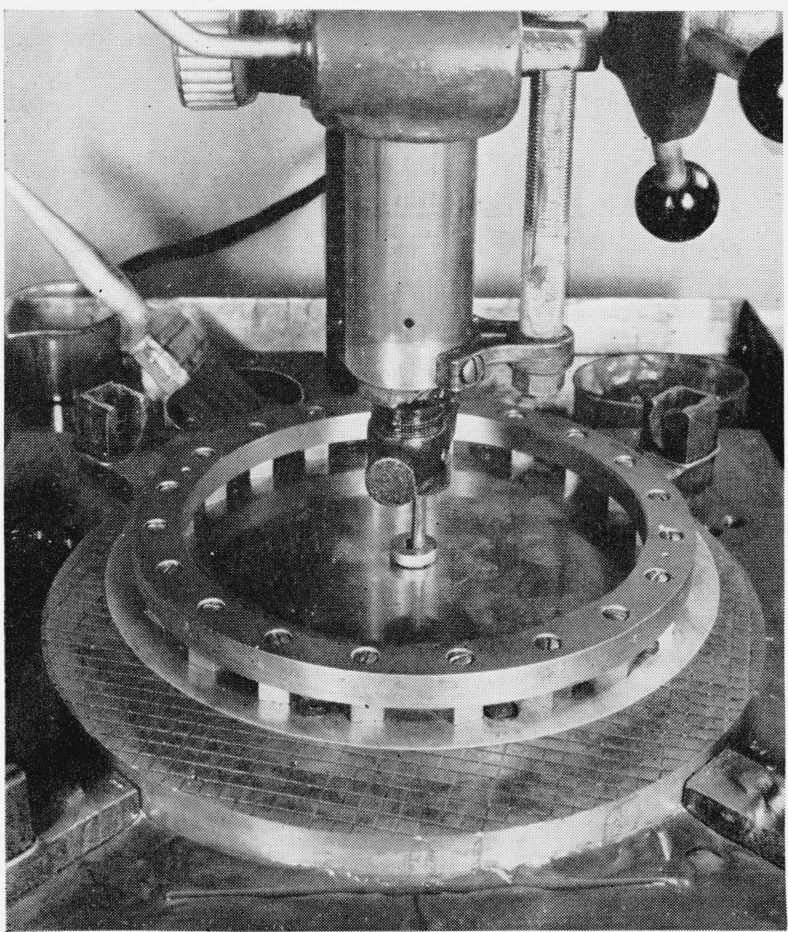

Figure 5. Pentagonal block apparatus.

Twenty pentagonal blocks, rigidly attached to a steel ring are shown resting on crystals (hidden) in the corresponding nest openings. Some of the springs that force the nest into close contact with the lap may be seen.

Results achieved by the use of the pentagonal block system were encouraging. Deviations from flatness were radial rather than wedge-like. The variation did not exceed $0.00004 \mathrm{in}$. and was, for the most part, concentrated in a rim-like bulge, the enclosed area being very nearly flat and parallel. BT crystals with a thickness of about $0.0025 \mathrm{in}$. and a fundamental frequency of $43 \mathrm{mc}$ were produced. Such crystals could be etched to even higher frequencies.

Experience with the pentagonal block system suggested two modified procedures. In one, the nest was eliminated; in the other, a thick nest with circular holes was used.

\section{Driven Ring Method}

The nest-less apparatus consisted of a flat ring upon which were mounted cylindrical blocks, each with an encircling ring or collar to retain the crystal (fig. 6). The collars and the under surfaces of the blocks were channeled to improve circulation of the abrasive mixture. An eccentric, acting through four rigid spokes, drove this assembly over the lower lap. Because the ring

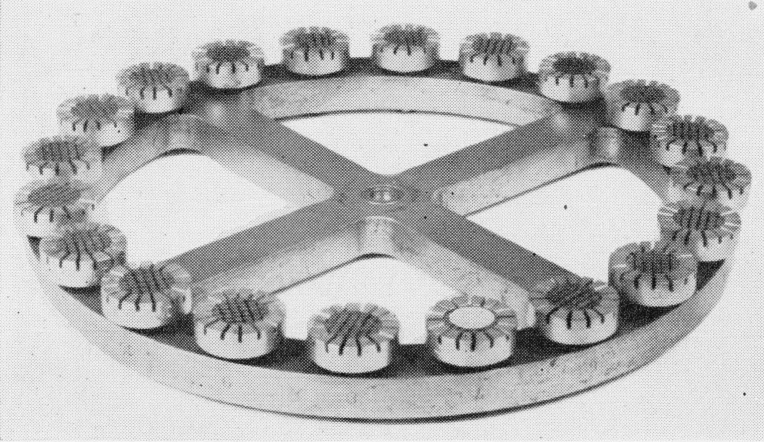

Figure 6. Dricen ring apparatus.

The apparatus has been inverted to show more clearly the relationship of the parts. A round crystal is shown resting on one of the lapping plugs and in contact with its confining collar. The collars have been slotted to permit freer circulation of abrasive.

and spokes were of thick steel, the load on the crystals was quite heavy. As a result, crystals began to break when approximately $0.004 \mathrm{in}$. thick. The investigation has been discontinued until a ring of lighter metal can be made.

\section{Ring and Thick Nest Method}

This modification made use of the same type of ring as above but without the spokes, since the ring was carried by the nest (fig. 7.) Cylindrical plugs were used without collars. Instead, holes were carefully drilled and reamed in a thick and rigid nest. Each hole was a sliding fit on any plug of the ring. (Indeed the main advantage of using the circular rather than the pentagonal

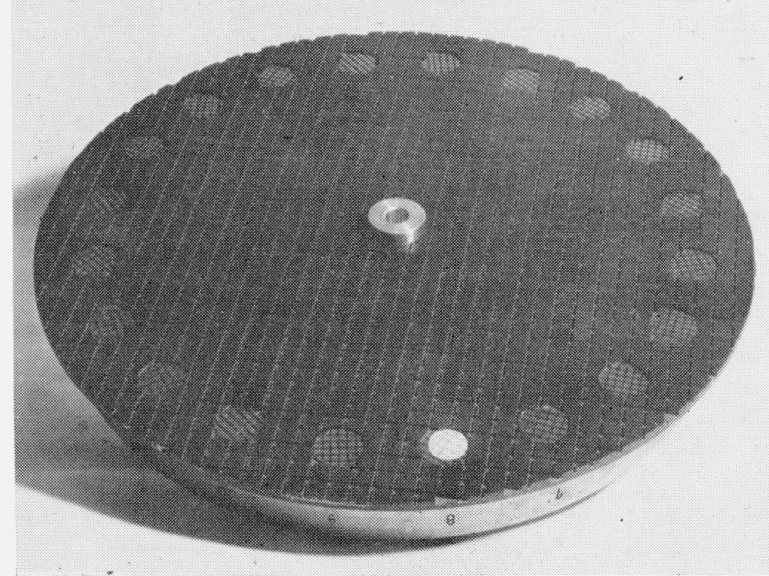

Figure 7. Ring and thick nest apparatus.

The apparatus is inverted to show the relationship of the parts. The crystal is resting on one of the round plugs and within the nest aperture that confines it during lapping. The plugs fit the apertures very closely to prevent the formation of rims on the crystals. 
openings lay in the ease with which apertures and plugs could be made to close tolerances). As in the pentagonal block method, the nest served both to retain the crystals and to adjust the pressure on them. This latter effect was accomplished by the use of small conical springs, the apex ends of which fitted tightly over studs located midway between the lapping blocks and the base ends of which pressed the nest into closer contact with the lapping plate. Changing the number of springs adjusted the pressure on the crystals, permitting heavier pressure and faster lapping on the thicker crystals and similarly, lower pressure and slower lapping on the thinner ones. Cross-channeling the under side of the nest with grooves $1 / 32$ in. wide by $1 / 16$ in. deep at $1 / 4-i n$. intervals allowed freer circulation of the abrasive and reduced adhesion between the lap and the nest.

Although this method is still under investigation, some conclusions have been drawn. Since the nests were thicker than those available commercially, the apertures maintained their size and shape much better than did the openings in the thin nests. Consequently, the clearance between each plug and its respective hole remained small and the resulting rim was much less pronounced. In the case of square crystals, the rimming was confined to the corners where its effect on performance appeared to be negligible. So far, crystals have been ground to a thickness of 0.002 in. by this method.

Although no definite limit was imposed on the final thickness of crystals-produced with the equipment last described, the hazards connected with removing the ring and nest in order to inspect crystals less than 0.002 in. thick made another method seem more desirable for the final stage of lapping.

\section{IX. "Inkwell" Method}

As mentioned earlier, a cylinder and plunger modification has been designed on the basis of experience with the square block and cell unit. In the improved form (fig. 8) the retaining cell consists of a circular aperture in a heavy-walled brass block, approximating an inkwell in size and shape. The conical shape is a compromise between height and stability, and the base is broad to maintain its true surface. The bottom part of the block is a detachable annular disk, the central

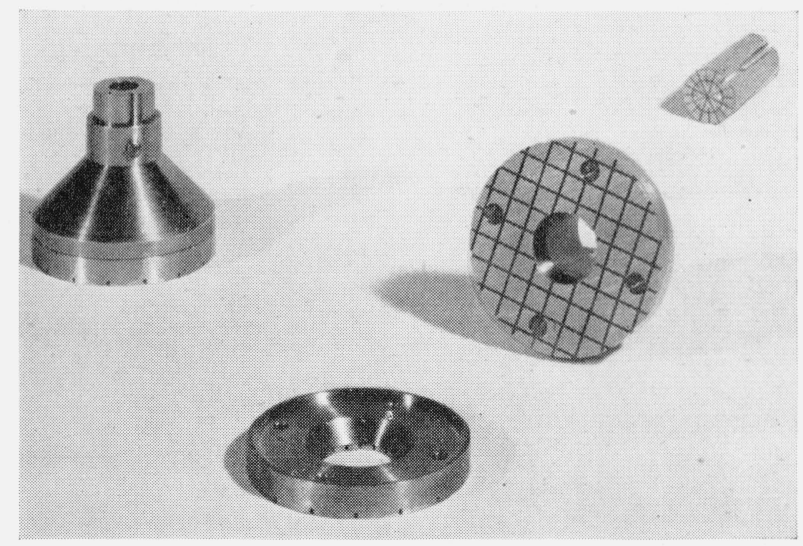

FIGURE 8. Inkwell apparatus-parts.

The keyed plunger, conical body, and annular disk are shown assembled at the left. In the foreground may be seen the upper surface, chamfer, and bore of the disk part. The lower surface and its relation to the bore is evident in the unit slightly to the right, while the plunger may be seen in the right background.

hole of which contains the crystal during lapping. The diameter of this hole is just enough larger than the plunger to prevent binding by the abrasive. For the same reason the cell is quite shallow. A recess on the upper block together with the chamfered upper surface of the disk prevents abrasive from reaching the more closely machined upper bore. Since the disk is detachable, it can be replaced easily when wear renders it unusable.

The cylinder bore is a smooth fit for $3 / 4$-in. drill rod. For lighter pressure, hollow aluminum plungers are being used. Close fit (especially in the upper bore) is essential to keep the plunger from wobbling. The plunger and block base are trued with respect to each other against the lap. A pin in the block wall engages a keyway in the upper part of the plunger thereby preventing its rotation while permitting vertical motion. In this manner the trued surfaces of the plunger and base remain parallel (except for the factor of prolonged wear). It is customary to true these surfaces at about 8-hour intervals. As in previous lapping units, the working surfaces are channeled to improve circulation of the abrasive and to reduce adhesion.

To operate, the loaded unit (cell block with plunger and crystal) is placed in one of the circular apertures of a heavy nest and carried over the lower lap in the usual curved path (fig. 9). Since the aperture is $0.015 \mathrm{in}$. larger than the cell block and the cell aperture is considerably larger than the enclosed crystal, all three - crystal, block, and 


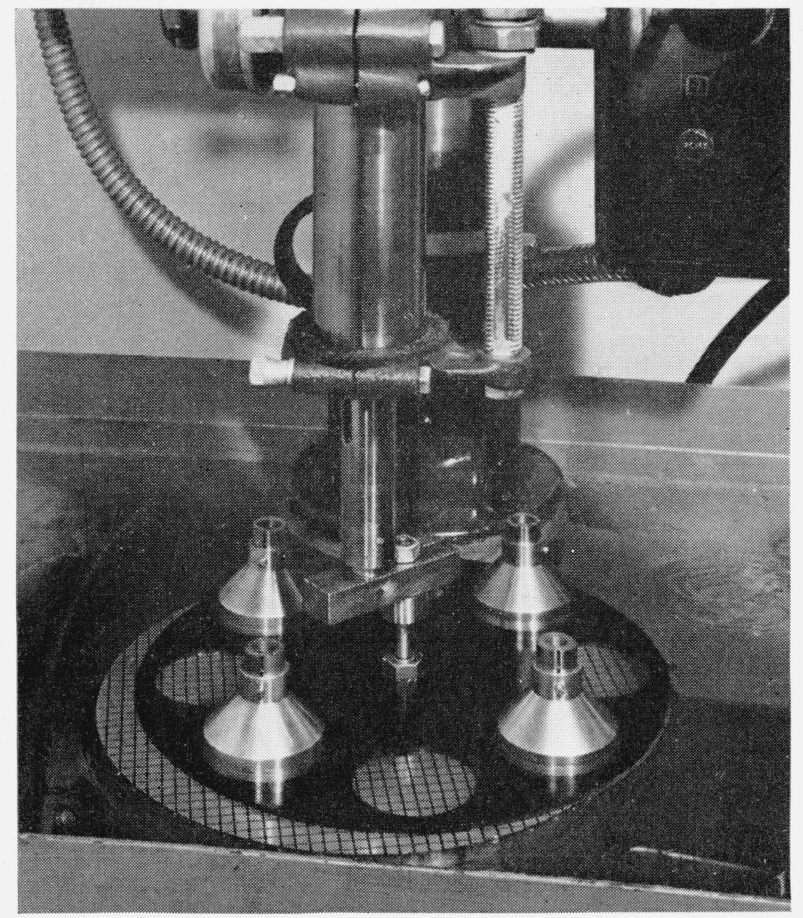

Figure 9. Lapping set-up with inkwell units.

The assembled units are shown ready to be driven over the lap in the usual manner.

nest-execute different eccentric motions. The present lap and nest can accommodate eight units. The crystal in any one of these units can be easily examined without disturbing any other crystal, a very useful feature.

Using this apparatus crystals have been lapped to about $0.001 \mathrm{in}$. with practically no breakage. The edges were smooth and free from radial cracks, and the faces were, in general, satisfactorily flat. Naturally crystals so thin are fragile and handling them in the usual routines of cleaning, measurement, plating, and mounting gives rise to important new problems. These problems, rather than the method of lapping, may set the ultimate limit for crystal thickness.

\section{Tall Plunger Method}

With the intention of regulating the piston wobble, a modification of the above unit has just been completed. In the new unit the plunger is taller and bears on rounded points rather than on a wall. Twelve screws with rounded ends serve as the bearing points, six being equally spaced around the piston near its top and another six

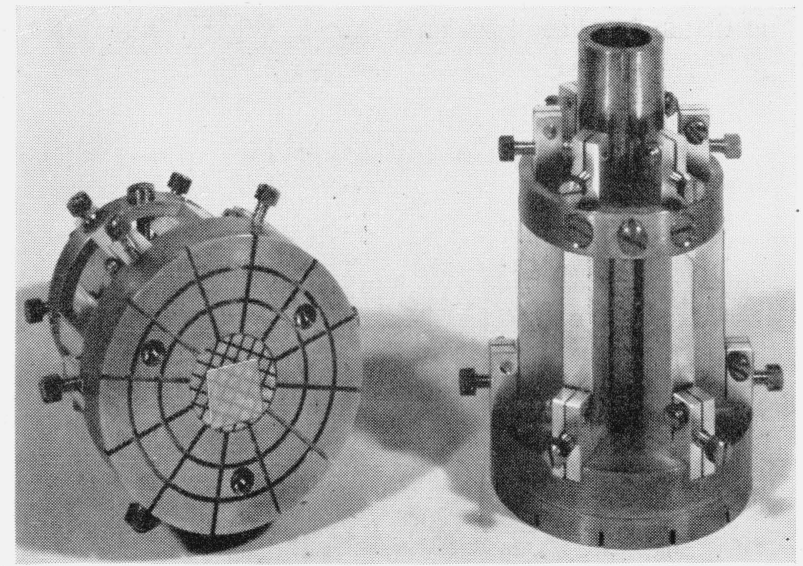

FiguRe 10. Tall plunger apparatus.

Bearing point screws replace the close fitting bore in this modification of the inkwell model. Slots in the uprights permit the transverse screws to lock the bearing point screws in position.

similarly spaced near the bottom (fig. 10). After these screws have been adjusted to permit sliding without lateral play, the transverse screws are tightened to maintain the fit. This modification has yet to be tested.

\section{Conclusion}

Of the ten methods described the last three were the most satisfactory. Although they have been developed primarily for the production of thin quartz oscillator plates they can be used for lapping a variety of mineral and ceramic materials. For example, plates of a ceramic dielectric have been lapped by the inkwell apparatus to 0.0015 -in . thickness. In such experimental work the individual plunger and block units are especially useful, since only a minimum of the test material is needed. As previously mentioned, a number of such units may be used with one driving nest to lap test specimens of various thicknesses simultaneously. However, since the driven nest with ring-mounted plugs accommodates more crystals on the same size lap it is more economical when many plates of the same thickness are to be made. Both methods have their special features, and it is likely that development will continue along two paths to provide machines that will better serve, on the one hand, for production of experimental or special orders, and on the other hand, for commercial quantities.

Washington, June 14, 1949. 